\title{
Is clipping the preferable technique to perform sympathicotomy? A retrospective study and review of the literature
}

\author{
Gregor J. Kocher • Anas Taha • Markus Ahler • \\ Ralph A. Schmid
}

Received: 13 May 2014 / Accepted: 9 September 2014 / Published online: 23 September 2014

(C) Springer-Verlag Berlin Heidelberg 2014

\begin{abstract}
Purpose Thoracoscopic sympathetic surgery is nowadays a broadly accepted technique in the treatment of primary hyperhidrosis as well as facial blushing. The objective of this study was to compare the two currently most commonly used methods for thoracic sympathicotomy: transection (ETS) and clipping (ETC.).

Methods This is a retrospective study on a total of 63 patients, who underwent rib-oriented sympathicotomy, either by transection $(n=36,57 \%)$ or by clipping $(n=27,43 \%)$. Moreover, the up-to-date international literature is reviewed concerning which level(s) of the sympathetic trunk should be addressed, depending on the patients underlying condition. Furthermore, the highly controversial topic of reversibility of sympathetic clipping is debated.

Results Our results confirm that clipping is at least as effective as transection of the sympathetic chain in the treatment of hyperhidrosis and facial blushing. Furthermore, the analysis of all larger studies on unclipping in humans shows a surprisingly high reported reversal rate between 48 and $77 \%$.

Conclusions Depending on the symptoms of the patient, different levels of the sympathetic chain should be addressed. When a higher rib level such as R2 is approached, which more likely will result in moderate to severe compensatory sweating, clipping should be preferred as it seems that this technique has indeed a potential for reversibility. As demonstrated, this method is at least as effective as an irreversible transection of the sympathetic chain.
\end{abstract}

Keywords Thoracoscopy · Sympathectomy $\cdot$ Hyperhidrosis · Facial blushing

G. J. Kocher $\cdot$ A. Taha $\cdot$ M. Ahler $\cdot$ R. A. Schmid $(\bowtie)$

Division of General Thoracic Surgery, University Hospital Bern, Freiburgstrasse, 3010 Bern, Switzerland

e-mail: ralph.schmid@insel.ch

\section{Introduction}

Palmar hyperhidrosis $(\mathrm{PH})$, axillary hyperhidrosis $(\mathrm{AH})$, and facial hyperhidrosis $(\mathrm{FH})$ as well as facial blushing $(\mathrm{FB})$ can cause severe physical, social, and psychological impairment, which ultimately may require surgical treatment. Nowadays, endoscopic thoracic sympathectomy is a well-established and effective treatment option for these patients, offering them a minimally invasive operation with high success rates and low morbidity, once conservative treatment options have failed. However, despite high success rates, patient satisfaction can be substantially adversely influenced by compensatory sweating (CS), sometimes to that extent that patients request a reversal of the procedure. While transection of the sympathetic chain (i.e. endoscopic thoracic sympathicotomy (ETS)) by means of an electrocautery or an ultrasonic device represents the method of choice in most centers, endoscopic thoracic sympathicotomy by means of clipping (ETC.) gains more and more acceptance, since it seems to lead to comparable results [1-3] but may in addition hold the potential for the reversal of the procedure [4].

In this retrospective study, we report our own results, comparing ETS and ETC. Furthermore, we performed a thorough review of the up-to-date international literature in order to find a consensus on the optimal level of the sympathetic chain, which should be approached, depending on the patients' underlying condition. Finally we analyzed all larger studies (including more than ten patients with a reversal procedure) focusing on the reversibility of ETC.

\section{Materials and methods}

Data were collected from a consecutive series of all patients who underwent video-assisted thoracic surgical sympathicotomy for idiopathic hyperhidrosis and/or facial 
blushing in our unit for general thoracic surgery at the University Hospital of Bern, Switzerland, from May 2004 through November 2012. The data was analyzed by reviewing the medical charts including a routinely performed outpatient visit within 2 weeks after the operation and a standardized telephone interview at the time of the data collection (July 2013). Informed consent for the study inclusion was obtained from all patients. The telephone questionnaire was created following the questionnaire provided by the International Society for Sympathetic Surgery (ISSS) [5]. Questions concerning the postoperative course mainly concentrated on the success of the procedure, complications, adverse side effects, (mainly CS) as well as overall patient satisfaction or even regret. The patients were asked to rate their preoperative condition as well as the effect achieved through the operation and the occurrence, location, and severity of any CS as follows: $1-$ no sweating, no disturbance/excellent result; 2-light to "normal" sweating/good result; 3 -increased sweating, disturbing but tolerable/no effect; and 4 - severe sweating, intolerable/ not satisfied. The main outcome variables were the success of the procedure (ratings 1-2), the disturbing/intolerable CS (ratings 3-4), and the overall patient satisfaction after surgery.

\section{Surgical technique}

Bilateral, rib-oriented sympathetic surgery was performed using two different approaches: Either the sympathetic chain was transected (ETS) through a single axillary trocar by means of a resectoscope with a monopolar cautery loop (Olympus Optical, Switzerland), or two to three ports were used and two titanium clips (Endo Clip ${ }^{\mathrm{TM}}$, COVIDIEN) were applied to the desired nerve level(s) (ETC.). In both patient groups, the tissue overlying the second rib was cauterized lateral to the sympathetic chain on a sector of at least $2 \mathrm{~cm}$ in order to include any accessory nerve fibers including the nerve of Kuntz. All procedures were performed under general anesthesia, involving the placement of a left-sided double-lumen endotracheal tube. The patients were placed in a half-sitting supine position with both arms elevated and supported to $90^{\circ}$. The procedure was usually started on the left side during single-lung ventilation, and after completion, the opposite side was approached in the same manner (i.e. one-stage procedure). Lungs were inflated under direct vision before closure of the portholes. No palmar temperature monitoring was used.

A postoperative routine chest radiograph was obtained in all patients after extubation in the recovery room to rule out significant pneumothorax and assess the correct level of the clips (if applicable).

\section{Data analysis}

Data are presented as mean \pm standard deviation. Statistical analysis was performed using the Fisher exact test and reporting two-tailed $p$ values. All $p$ values less than 0.05 were considered statistically significant.

\section{Results}

During the study period, a total of 63 patients were enrolled. The main patient's characteristics are listed in Table 1. All patients had been evaluated for secondary causes of hyperhidrosis and/or facial blushing, and conservative treatment options have been exhausted in all cases before consideration for sympathectomy. Previous non-operational treatment consisted of topic agents (aluminum chloride) in all hyperhidrosis cases, iontophoresis in $35 \%(n=22)$, botulinum toxin A injections in $19 \%(n=12)$, and oral medications in $22 \%(n=14)$ of patients (moxonidine in 13 and Trandate in 1). Nonoperational treatment was either only short lasting and/ or not sufficiently effective or associated with unsustainable side effects (mainly tiredness and exercise intolerance in patients receiving the aforementioned oral medication). All patients admitted for surgery rated at least one of their symptoms a 4 on the aforementioned scale. Only $24 \%(n=15)$ of patients reported to suffer from excessive sweating in only one single region.

Of the 63 patients, $36(57 \%)$ underwent ETS by means of a resectoscope, while the remaining 27 (43\%) patients underwent ETC. The main results are listed in Table 2. The choice of the particular surgical technique was mainly based on chronological order: Until July 2006, all patients routinely underwent ETS of the levels R2-4. After that time, the technique was adapted to $\mathrm{R} 2+3$. Also the performance of the ETC. was based on chronological order, since we first introduced the procedure for the treatment of patients suffering from facial blushing (also if in combination with palmar hyperhidrosis) in 2009. With the increasing popularity of this technique and encouraged by our own results, we then completely switched to this technique for all sympathetic operations in June 2011.

Table 1 Patient's characteristics and symptoms

\begin{tabular}{ll}
\hline Patient characteristics & Percentage (number $n=63$ ) \\
\hline Gender & $59 \%$ female $(37)$ \\
Age in years (mean $\pm \mathrm{SD})$ & $30 \pm 11.8$ \\
Facial blushing $(\mathrm{FB})$ & $30 \%(19)$ \\
Facial hyperhidrosis $(\mathrm{FH})$ & $25 \%(16)$ \\
Palmar hyperhidrosis $(\mathrm{PH})$ & $76 \%(48)$ \\
Axillary hyperhidrosis $(\mathrm{AH})$ & $44 \%(28)$ \\
Plantar hyperhidrosis & $17 \%(11)$ \\
\hline
\end{tabular}


Table 2 Operative techniques and results

\begin{tabular}{|c|c|c|c|c|}
\hline Variable & Total & ETS (transection) & ETC. (clipping) & $p$ value \\
\hline Number $(n)$ & 63 & $57 \%(36)$ & $43 \%(27)$ & \\
\hline $\mathrm{R} 2$ & & $2.8 \%(1)$ & $37 \%(10)$ & \\
\hline $\mathrm{R} 2 / \mathrm{R} 3$ & & $58.3 \%(21)$ & $63 \%(17)$ & \\
\hline R2/3/4 (until 7/2006) & & $38.8 \%(14)$ & - & \\
\hline Follow-up (in months) & $57.8 \pm 31.3$ & & & \\
\hline \multicolumn{5}{|l|}{ Success rates (rating 1-2) } \\
\hline FB $(n=19)$ & $81 \%$ & $83 \%(10 / 12)$ & $75 \%(3 / 4)$ & 1.0 \\
\hline FH $(n=16)$ & $94 \%$ & $100 \%(12 / 12)$ & $75 \%(3 / 4)$ & 0.25 \\
\hline $\mathrm{PH}(n=48)$ & $98 \%$ & $100 \%(14 / 14)$ & $96 \%(26 / 27)$ & 1.0 \\
\hline $\mathrm{AH}(n=28)$ & $38 \%$ & $29 \%(2 / 7)$ & $41 \%(7 / 17)$ & 0.67 \\
\hline \multicolumn{5}{|l|}{ Severe CS (rating 4) } \\
\hline $\mathrm{R} 2$ & & $100 \%(1 / 1)$ & $30 \%(3 / 9)$ & 0.4 \\
\hline $\mathrm{R} 2 / \mathrm{R} 3$ & & $16 \%(3 / 18)$ & $31 \%(5 / 16)$ & 0.43 \\
\hline $\mathrm{R} 2 / 3 / 4$ & & $25 \%(3 / 12)$ & - & - \\
\hline Total & $27 \%(15 / 56)$ & $23 \%(7 / 31)$ & $32 \%(8 / 25)$ & 0.55 \\
\hline Moderate to severe CS (rating 3-4) & $82.1 \%(46 / 56)$ & & & \\
\hline Overall satisfaction & $94.6 \%(53 / 56)$ & $90.3 \%(28 / 31)$ & $100 \%(25 / 25)$ & 0.24 \\
\hline
\end{tabular}

\section{Hospital stay}

Before the year 2010, patients stayed in the hospital overnight and were usually discharged on the first postoperative day (except for one patient - see complications). Since the beginning of 2010, all patients were routinely discharged on the same day, about 4-5 h after surgery.

\section{Follow-up}

During July 2013, all patients were approached by telephone questionnaire with a mean follow-up period of $57.8 \pm$ 31.3 months (interval 5-117 months). Response rate for the telephone questionnaire was 56 out of 63 patients $(88.9 \%)$. Of the seven patients not accessible for follow-up, one had died of metastatic ovarial cancer and two patients reported to be still satisfied with the result but refused to answer the whole questionnaire in detail. The remaining four patients could not be located at all.

\section{Outcome}

Success rates (including good (2) and excellent (1) results) were highest for the treatment of $\mathrm{PH}$ with $98 \%$ (40 of 41 patients), followed by facial hyperhidrosis with $94 \%$ (15 out of 16 patients) and facial blushing with $81 \%$ (13 of 16 patients). All three failures in the treatment of facial blushing were attributable to technical errors (cutting/clipping one level too low). Insufficient treatment was observed for $\mathrm{AH}$ with improvement in only $38 \%$ of patients ( 9 of 24 patients).
Despite a relatively high rate of moderate $(55 \%, 31 / 56)$ or even severe $(27 \%, 15 / 56$ patients) CS, mainly occurring in the back and the trunk region, the overall satisfaction rate for all procedures was $94.6 \%$.

There was no significant statistical difference between the ETS and the ETC. group concerning the success rate, as well as the CS rate. Furthermore, no statistically significant difference in CS rates between the R2, R2/3, and R2/3/4 group could be detected.

Failure (i.e. no effect) of the procedure was seen in three patients. Two of them were already mentioned above, both undergoing clipping of R3 instead of R2, which was revealed on postoperative chest $\mathrm{x}$-rays and resulted in the resolution of their predominant $\mathrm{PH}$ but without any effect on their concomitant FB. The third patient suffered from FB, $\mathrm{PH}$, and $\mathrm{AH}$ and underwent ETS of R2+3, whereupon palmar and axillary hyperhidrosis disappeared, while facial blushing persisted. Very likely the same problem as in the two ETC. patients also occurred in this patient. Both ETC. patients were nevertheless satisfied with the result (since $\mathrm{PH}$ was their main reason for surgery) and did not want to undergo any reoperation. The ETS patient after all, despite successful treatment of $\mathrm{PH}$ and $\mathrm{AH}$, expressed regret, since he suffered from severe CS. Also he declined a reoperation for approaching R2 as well, since he was afraid that CS would even increase with that.

\section{Complications/reoperations}

Surgical complications were very rare. Only one patient had to stay in the hospital longer than intended due to unilateral air leak after intraoperative adhesiolysis, with chest drain 
duration of 5 days. No severe complications like Horner's syndrome or hemothorax occurred.

Two patients were reoperated: Patient 1 underwent ETC. of $\mathrm{R} 2 / 3$ for palmar and axillary hyperhidrosis and was reoperated (additional ETC. of R4) upon his own urgent request 2 months later due to an insufficient effect on axillary hyperhidrosis. After an additional clipping of R4, the axillary hyperhidrosis slightly improved (from 4 - severe to 3 -tolerable). The second patient underwent ETS of R2/3 for facial hyperhidrosis and was reoperated 1 year later on the right side, due to unilateral recurrence of facial sweating. But even after reoperation (right re-ETS R $2 / 3$ including any accessory fibers), the asymmetry persisted and the patient finally regretted having undergone the operation in the first place.

As mentioned above, in total only three patients (4.8\%), all undergoing ETS R2/3, expressed regret. No patient in the ETC. group regretted the operation, consequently none of these patients requested reversal of the procedure.

\section{Discussion}

Thoracoscopic sympathectomy has been shown to be the most efficacious and durable treatment option for palmary hyperhidrosis. Furthermore, facial blushing, as well as facial sweating and even axillary sweating, can be treated with this method with high success rates in selected patients. The main concerns, when discussing the optimal technique for sympathetic surgery, are mainly the success of the operation on one hand and associated adverse effects, such as compensatory sweating, on the other. Furthermore, the potential for reversal in case of an unsatisfactory postoperative result would be highly desirable.

As shown by our own results and confirmed by other authors, clipping is at least as effective as transection of the sympathetic chain. Unfortunately, not only the success rates but also the rate of compensatory sweating seems to be the same for both methods [1-3]. Since CS is not only the most common cause for patient dissatisfaction but also the most important reason for patients to request the reversal of the procedure [6,7], efforts have been made to avoid or at least decrease CS.
As described by several authors $[8,9]$, there is evidence that approaching multiple levels of the sympathetic chain seems to result in increased rates of severe CS. Furthermore, it has been shown that the involvement of the R2 segment seems to have an impact on CS rates and severity $[10,11]$. The optimal level to be addressed, depending on the patients' underlying condition, is still a matter of debate in the international literature, but evidence is increasing that for facial blushing, approaching the R2 level seems to be mandatory. By approaching R3 only, facial sweating is reported to improve, by at the same time conserving a normal moisture of the face and decreasing the rate of $\mathrm{CS}$, which consequently resulted in better patient satisfaction. For the treatment of palmar hyperhidrosis, "R4 only" is reported to result in high success rates, minimal CS, and furthermore the advantage of less over dryness of the hands [3, 12-15]. For axillary sweating, approaching R4 or even R5 has been suggested as a treatment option, but considering the possible side effects of the ETS/ETC. procedure, Heidemann and Licht [16] have shown that for patients suffering from isolated $\mathrm{AH}$, a local treatment by subcutaneous liposuction/curettage might be more suitable, offering them a local therapeutic option under local anesthesia with minimal side effects and complication rates. However, when treating a combination of $\mathrm{PH}$ and $\mathrm{AH}$, R4 seems to be the optimal target level for both conditions.

Besides the aforementioned technical details of the particular operative procedure, also patient characteristics, especially BMI $(>28)$ and older age, were shown to adversely influence the rate and severity of $\mathrm{CS}$, which should be taken into account when informing and selecting patients for ETS/ETC. [9].

Another important point is the issue of reversibility of the operation, especially considering that not only CS but also other adverse events or complications, such as symptomatic bradycardia in combination with exercise intolerance or the occurrence of a Horner' syndrome, could only be treated with reversal of the procedure. Lin et al. [1] were the first to report the application of clips to the sympathetic chain without the need for its transection. Not only were the success rates comparable to other more invasive techniques [2] but also in case of reversal there would be no need for a complex reconstruction operation, but rather a comparatively simple thoracoscopic removal of the clips.
Table 3 Results of unclipping studies

$N / A$ not available

\begin{tabular}{llrlll}
\hline Author (year) & $\begin{array}{l}\text { Total number } \\
\text { of patients }\end{array}$ & $\begin{array}{l}\text { Unclipping } \\
\text { procedures }\end{array}$ & $\begin{array}{l}\text { Targeted level } \\
\text { R2 } \pm \text { R3 }\end{array}$ & Success rate & $\begin{array}{l}\text { Mean interval } \\
\text { to reversal }\end{array}$ \\
\hline Sugimura et al. (2009) [20] & 727 & $4.7 \%(34)$ & $82 \%(28)$ & $48 \%(15)$ & 11 months \\
Kang et al. (2008) [4] & 116 & $12.9 \%(15)$ & $87 \%(13)$ & $60 \%(9)$ & 29.5 days \\
Chou et al. (2006) [19] & 464 & $2.8 \%(13)$ & $92 \%(12)$ & $77 \%(10)$ & N/A \\
$\begin{array}{l}\text { Reisfeld et al. (2006/09) } \\
\quad 111,21]\end{array}$ & 1274 & $2 \%(25)$ & $88 \%(22)$ & $52 \%(13)$ & N/A \\
$\quad$ & & & & \\
\end{tabular}


What sounded like a promising concept in theory first seemed to be not that reproducible in clinical practice. Loscertales et al. [17], for example, showed in a swine model that Wallerian degeneration is a common finding already 10 days after clipping and that there was no evidence of nerve regeneration at 10 or 20 days after clip removal. On the other hand, a recently performed study by Bayram et al. [18], investigating clipping of the sympathetic chain and clip removal in goats, presented on the 27th EACTS - meeting in Vienna (October 5-9, 2013), clearly supported the possibility of nerve regeneration after unclipping. More animal studies on this issue are avaited.

In order to collect all the relevant existing data on clip removal in human patients, we performed a Medline database search, looking for reports, which investigate the results of reversal of the procedure in humans and contained at least ten patients [4, 11, 19-21]. The data are presented in Table 3 and obviously, indeed a success rate of 48-77\% was reported, whereas success was defined as a decrease in CS and with that an increase in patient satisfaction. As discussed before, also in these studies, higher rates of CS and consequently a higher rate of requested reversal procedures was observed when higher levels as R2 and R3 were targeted during the initial operation.

As underlined by the existing animal studies and also suggested by the aforementioned authors investigating the reversal procedure in humans, clip removal should be performed as early as possible in order to hopefully achieve better results, since the nerve tissue might have a better potential for regeneration. As furthermore discussed by Kang et al. [4], not only the time to removal of the clips but also the clamping power used for the clip application in the first place might be an important factor influencing nerve recovery after unclipping and with that the chances of reversibility. Since it is difficult to estimate the correct amount of clamping power needed to interrupt the nerve impulses, without irreversibly damaging the nerve itself, they suggested the use of a finger temperature monitoring, indicating the right amount of compression power.

Main limitations of this study include its retrospective nature, as well as the fact that the study is not randomized and includes only a relatively small number of patients compared to other studies. Nevertheless, this study was performed at a single institution, involving patients with similar demographic data in both groups and involving the same team of surgeons, with its corresponding impact on patient selection and standardized operative technique, and thus making the results of the two groups highly comparable.

In conclusion, our study confirms that clipping is at least as effective as transection of the sympathetic chain in the treatment of hyperhidrosis and facial blushing, which is also underlined by other authors. Furthermore, the up-to-date international literature provides increasing evidence that, depending on the symptoms of the patients, different and especially lower levels of the sympathetic chain should be approached in order to reduce the risk for severe CS: R2 for facial blushing, R3 for facial sweating, and R4 for palmary and/or axillary hyperhidrosis. Assuming that there is truly a possibility for reversal of the ETC. procedure, as shown in our analysis of several studies, we believe that this technique should at least be preferred whenever a more cranial level like $\mathrm{R} 2$ is approached, which is more likely to result in higher rates and severity of CS.

In the future, intraoperative monitoring (i.e. by means of a temperature probe, sudometry, etc.) may help not only in identifying the optimal level for ETC. but also in determining the amount of force needed while clamping/clipping the sympathetic chain. This may not only ensure constantly high success rates of the procedure but also hopefully reduce the rate and intensity of CS and furthermore render a more promising and reliable reversal of the operation.

\section{Conflicts of interest None.}

Authors' contributions Kocher and Taha were responsible for the study conception and design and acquisition, analysis, and interpretation of data. Ahler was also responsible for the acquisition, analysis, and interpretation of data. Kocher and Schmid were responsible for the drafting of the manuscript, and Schmid was also responsible for the study conception and design, analysis and interpretation of data, and critical revision of the manuscript.

\section{References}

1. Lin CC, Mo LR, Lee LS, Ng SM, Hwang MH (1998) Thoracoscopic T2 sympathetic block by clipping: a better and reversible operation for treatment of hyperhidrosis palmaris - experience with 326 patients. Eur J Surg 164:13-16

2. Reisfeld R, Nguyen R, Pnini A (2002) Endoscopic thoracic sympathectomy for hyperhidrosis: experience with both cauterization and clamping methods. Surg Laparosc Endosc Percutan Tech 12:255-267

3. Cerfolio RJ, De Campos JR, Bryant AS, Connery CP, Miller DL, DeCamp MM et al (2011) The Society of Thoracic Surgeons expert consensus for the surgical treatment of hyperhidrosis. Ann Thorac Surg 91(5):1642-1648

4. Kang CW, Choi SY, Moon SW, Cho DG, Kwon JB, Sim SB et al (2008) Short-term and intermediate-term results after unclipping: what happened to primary hyperhidrosis and truncal reflex sweating after unclipping in patients who underwent endoscopic thoracic sympathetic clamping? Surg Laparosc Endosc Percutan Tech 18(5): 469-473

5. Questionnaire "Quality of Life Disturbances Due to Palmar Hyperhidrosis". Available at http://www.isss.net/recommendations. html. Accessed Jun 2013

6. Gossot D, Toledo L, Fritsch S, Célérier M (1997) Thoracoscopic sympathectomy for upper limb hyperhidrosis: looking for the right operation. Ann Thorac Surg 64:975-978

7. Lin CC, Telaranta T (2001) Lin-Telaranta classification: the importance of different procedures for different indications in sympathetic surgery. Ann Chir Gynaecol 90:161-166 
8. Licht PB, Pilegaard HK (2004) Severity of compensatory sweating after thoracoscopic sympathectomy. Ann Thorac Surg 78:427-431

9. Miller DL, Bryant AS, Force SD, Miller JI Jr (2009) Effect of sympathectomy level on the incidence of compensatory hyperhidrosis after sympathectomy for palmar hyperhidrosis. J Thorac Cardiovasc Surg 138(3):581-585

10. Weksler B, Blaine G, Souza ZB, Gavina R (2009) Transection of more than one sympathetic chain ganglion for hyperhidrosis increases the severity of compensatory hyperhidrosis and decreases patient satisfaction. J Surg Res 156(1):110-115

11. Reisfeld R (2006) Sympathectomy for hyperhidrosis: should we place the clips at T2-3 or T3-T4? Clin Auton Res 16:384-389

12. Weksler B, Luketich JD, Shende MR (2008) Endoscopic thoracic sympathectomy: at what level should you perform surgery? Thorac Surg Clin 18(2):183-191

13. Chang YT, Li HP, Lee JY, Lin PJ, Lin CC, Kao EL et al (2007) Treatment of palmar hyperhidrosis: $\mathrm{T}(4)$ level compared with $\mathrm{T}(3)$ and T(2). Ann Surg 246(2):330-336

14. Yang J, Tan JJ, Ye GL, Gu WQ, Wang J, Liu YG (2007) T3/T4 thoracic sympathictomy and compensatory sweating in treatment of palmar hyperhidrosis. Chin Med J (Engl) 120(18):1574-1577

15. Liu YG, Yang J, Wang J, Liu J, Li JF, Jiang GC et al (2006) A comparison of $\mathrm{T} 3$ sympathectomy versus $\mathrm{T} 4$ sympathectomy in the treatment of primary palmar hyperhidrosis. Zhonghua Yi Xue Za Zhi 86(33):2318-2320

16. Heidemann E, Licht PB (2013) A comparative study of thoracoscopic sympathicotomy versus local surgical treatment for axillary hyperhidrosis. Ann Thorac Surg 95(1):264-268

17. Loscertales J, Congregado M, Jimenez-Merchan R, Gallardo G, Trivino A, Moreno S et al (2012) Sympathetic chain clipping for hyperhidrosis is not a reversible procedure. Surg Endosc 26(5):12581263

18. Bayram A, Salci H, Peker S, Melek H, Gebitekin C. Can sympathetic nerve damage be reversed? 27th annual meeting of the European Association for Cardio-Thoracic Surgery, October 5-9 2013, Vienna, Austria

19. Chou SH, Kao EL, Lin CC, Chang YT, Huang MF (2006) The importance of classification in sympathetic surgery and a proposal mechanism for compensatory hyperhidrosis: experience with 464 cases. Surg Endosc 20:1749-1753

20. Sugimura H, Spratt EH, Compeau CG, Kattail D, Shargall Y (2009) Thoracoscopic sympathetic clipping for hyperhidrosis: long-term results and reversibility. J Thorac Cardiovasc Surg 137(6):1370-1376

21. Reisfeld R. Sympathectomy reversal. Clamping vs nerve graft. 8th international symposium on sympathetic surgery (ISSS), March 2527, 2009. New York, USA 